\title{
Timing individuals in a group using a computer-generated code sequence
}

\author{
WILLIAM R. FEENEY \\ San Diego State University, San Diego, Cailfornia 92115
}

\begin{abstract}
A method of timing individual subjects in any size group is described. Using a standard movie projector, a specially produced $16-\mathrm{mm}$ film projects codes composed of three letters. Each code remains on the screen for $2 / 3$ sec. Subjects are asked to record the code they see after completing each experimental task. Computer programs, written in BASIC, generate the 1,000 codes used in the film, edit the subject-recorded codes, and translate the codes into time measures. The code sequence on the film is designed: (1) to discourage subjects from attempting to calculate their own interval times, (2) to catch false codes and recording errors, and (3) to allow the experimenter who knows the structure of the codes to easily translate individual codes into time.
\end{abstract}

Sidowski (1966, p. 19) notes that, for certain experimental conditions, it might be advantageous to run large groups of subjects simultaneously. Since problems of stimulus presentation, response recording, and so on, are involved and difficult to control, he suggests the need for a display to permit individual timing measures in large groups. I have devised such a timing display, a 16-mm film that, when run on a standard projector, provides a timing method suitable for research. Although the method is not without some difficulties, it does serve the purpose of running large groups of subjects simultaneously.

The timing method has been used in experiments where the subject's individual time to perform short paper-and-pencil tasks is measured. The experiments were conducted in a classroom-like setting with a projector showing the film on a screen at the front of the room. In addition to written responses to experimental tasks, the subjects wrote down a three-letter code that was shown at the time they looked at the screen. By recording unique codes, the time taken to do a task can be determined. The three-letter codes change every $2 / 3 \mathrm{sec}$, and although they appear to be random, the sequence of codes do follow a pattern. This pattern, known to the experimenter but not to the subjects, helps detect subject copying errors and wild guesses.

Letter codes are used to discourage a subject from thinking of the time he takes to perform a task. Digits instead of letters could have been used, but with up to 26 individual letters to choose from instead of only 10 digits, a shorter, alphabetic code was devised.

\section{THE TIMING FILM}

Since the codes are on the screen for only a few moments at a time, the film appears to be a random three-letter grouping. Table 1 shows the first 30 codes on the film, counting from 0 to 29 of the 1,000 codes
Table 1

The First 30 of 1,000 Codes Used

\begin{tabular}{cccccc}
\hline Value & Code & Value & Code & Value & Code \\
\hline 0 & CLM & 10 & CKM & 20 & CIM \\
1 & EML & 11 & EMK & 21 & EMI \\
2 & BLM & 12 & BKM & 22 & BIM \\
3 & VML & 13 & VMK & 23 & VMI \\
4 & XLM & 14 & XKM & 24 & XIM \\
5 & TML & 15 & TMK & 25 & TMI \\
6 & LLM & 16 & LKM & 26 & LIM \\
7 & FML & 17 & FMK & 27 & FMI \\
8 & KLM & 18 & KKM & 28 & KIM \\
9 & DML & 19 & DMK & 29 & DMI \\
\hline
\end{tabular}

used in the film. A short computer program generates the 1,000 codes. When the film was made, 18 frames of each code were shot so the film would project at $2 / 3 \mathrm{sec}$ for each code at sound speed ( $24 \mathrm{frames} / \mathrm{sec})$ and $1 \mathrm{sec}$ at silent speed ( 18 frames/sec).

The algorithm used to produce the sequence of codes is designed to: (1) catch false codes containing invalid letters, (2) catch correctly recorded codes that are out of sequence, and (3) catch codes recorded with transposed characters.

To accomplish this, the code development algorithm has the following features: First, only 20 of the 26 letters of the alphabet are used $(G, J, Q, U, Y$, and $Z$ are omitted). Since it is impossible to detect by observation which letters are not used, inclusion of an omitted letter in a subject-written code makes it invalid.

Second, the order of appearance of each letter in a code is specified. The first letter in each code is randomly chosen for groups of 10 codes and is repeated every 10 codes. The last two letters of each code remain the same for each group of 10 , but alternate positions. The letters of the codes count as in the decimal number system, but to make instantaneous visual translation of the codes into numbers more difficult, the letters are in reverse order from the usual digit order in a number. 
Table 2

Letter Values Used in Generating the Code Sequence by Decimal Position

\begin{tabular}{ccccccc}
\multicolumn{2}{c}{$10^{\circ}$} & & \multicolumn{2}{c}{$10^{1}$} & \multicolumn{2}{c}{$10^{2}$} \\
\cline { 5 - 6 } Letter & Value & Letter & Value & Letter & Value \\
\hline C & 0 & L & 00 & M & 000 \\
E & 1 & K & 10 & N & 100 \\
B & 2 & I & 20 & O & 200 \\
V & 3 & H & 30 & P & 300 \\
X & 4 & F & 40 & R & 400 \\
T & 5 & E & 50 & S & 500 \\
L & 6 & D & 60 & T & 600 \\
F & 7 & C & 70 & V & 700 \\
K & 8 & B & 80 & W & 800 \\
D & 9 & A & 90 & X & 900 \\
\hline
\end{tabular}

That is, the three letters of an individual code have the order $10^{0}, 10^{1}, 10^{2}$, rather than $10^{2}, 10^{1}, 10^{\circ}$. A further switch makes visual translation even more difficult for the subject. For even-number representations, the sequence is always $10^{0}, 10^{1}, 10^{2}$, but for odd numbers, the order is changed to $10^{\circ}, 10^{2}, 10^{1}$.

All of the letters used in the codes and their numerical equivalents are shown in Table 2. With this table, it is possible to develop the codes used to produce the film and to translate a subject-written code into elapsed times.

There appear to be $26^{3}$, or 17,576 , possible threeletter combinations for the code sequence. Since only 1,000 of these are used in the film, the chance of a subject's guessing a correct code is $1: 17.5$.

A computer code-to-time program translates the three-letter codes written by the subjects into times. This program produces an error notice for (1) a threeletter code containing an invalid letter, (2) a code whose letters are valid but not in a correct sequence within a code, and (3) a code that is valid and has a correct within-code sequence, but that produces overlapping interval times.

\section{EXPERIENCE WITH THE TIMING FILM}

To date, the film has been used for timing five experiments in which the amount of interval time was the critical measure. The time for the subject to look at the screen and to record the code is treated as a constant time added to each task and is not subtracted from the task time during data analysis. This time, determined in a separate experiment, averaged $3 \mathrm{sec}$ with a standard deviation of $1.1 \mathrm{sec}$. Results from this experiment also showed no significant learning effect for subjects recording 13 codes for the 12 tasks required of each experiment.

Another experiment assessed what effect, if any, recording codes after each task had on subject preference. Subjects were given two sets of similar tasks. One required subjects to record their own times using the timing film, and the other contained sets of equivalent tasks on which subjects were timed individually. There was a correlation between the two sets of scores $[\mathrm{t}(20)=6.41, \mathrm{p}=.001]$, suggesting that the recording performed between tasks did not disrupt the subjects' ability to perform the tasks.

To acquaint subjects with the timing film and code recording, $.5 \mathrm{~min}$ of film was shown and then stopped. When the first experimental task started, the projector was turned on and the subjects recorded the first code shown. This code was used as the start time for the first trial. The experiment used tasks that did not exceed the 10-min length of the timing film.

For longer experiments, a film composed of splicedtogether copies of the 10-min film can be used, as long as each task is less than half the total length of the film, so it is possible to judge which 10-min segment the subject is in. Another approach is to run the film at silent speed, making the intervals $1 \mathrm{sec}$ and total showing time $15 \mathrm{~min}$. Another alternative for very long tasks is to show the film on a frame-by-frame basis using a variable speed projector. Each frame can then be shown as long as desired, providing a total timing capability of any desired length.

Less than $3 \%$ of the codes are incorrectly recorded by the subjects. At times, the last two letters of the code are transposed; rarely is the first character wrong. Sometimes an invalid letter is recorded that resembles a letter in the code (e.g., a "J" for an "I"). The code translation program notes all subject-recording errors. However, the experimenter is required to correct any invalid or incorrect codes before the program calculates the times. The intended code can usually be reconstructed by reversing two letters, interpreting a scribbled letter, or trying a look-alike letter (e.g., an " $\mathrm{H}$ " for an "N"). Rarely do data have to be discarded because of incorrect codes being unrepairable.

Subject tasks taking less than $4 \mathrm{sec}$ are suspect, and these subjects' data are discarded. A subject's taking so little time indicates he copied codes in quick succession without working on the tasks between recording the codes.

Figure 1 shows the computer output of the code-totime translation program for a single subject. The experiment that produced these numbers required the subject to answer three questions while referring to four successively presented displays. Multiple-choice questions were used, requiring subjects to perform a simple motor task or an arithmetic calculation. The 12

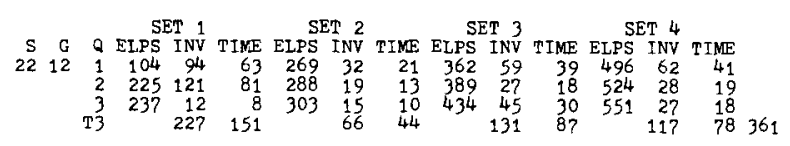

Figure 1. Example output from the code-to-time translation program for one subject performing 12 experimental tasks as four sets of 3 tasks. 
numbers resulting from the 13 recorded codes are shown under the column headed "ELPS" (i.e., elapsed time). The next column "INV" (short for interval time), is the amount of time the subject took to do each separate task. Both the elapsed and interval times are in units of $2 / 3 \mathrm{sec}$, the time each code stayed on the screen. The "TIME" column is the interval time in seconds the subject took to complete the task. The numbers in row "T3" are total times in seconds.
No special equipment is used in projecting the timing film. The tolerances of our standard $16-\mathrm{mm}$ movie projectors are $\pm 2 \%$.

\section{REFERENCES}

SIDowski, J. B. Experimental methods and instrumentation in psychology. New York: McGraw-Hill, 1966.

(Received for publication August 30, 1977; revision accepted May 5,1978 .) 